\title{
Gold nanoparticle-based enhanced lateral flow immunoassay for detection of Cronobacter sakazakii in powdered infant formula
}

\author{
Ruili Pan, ${ }^{1}$ Yujun Jiang, ${ }^{1}$ Luhong Sun, Rui Wang, Kejin Zhuang, Yueming Zhao, Hui Wang, Md. Aslam Ali, \\ Honghua $\mathrm{Xu}$, and Chaoxin Man ${ }^{2}$ \\ Key Laboratory of Dairy Science, Ministry of Education, Department of Food Science, Northeast Agricultural University, Harbin, China, 150030
}

\begin{abstract}
Cronobacter sakazakii is an opportunistic foodborne pathogen that can infect newborns through powdered infant formula (PIF). In this study, we developed a novel enhanced lateral flow immunoassay (LFA) with enhanced sensitivity for detection of $C$. sakazakii in PIF by the naked eye. The proposed strategy for signal enhancement of the traditional LFA used concentrated gold nanoparticles (AuNP) as the enhancer to conjugate with capture antibodies, which could increase the immobilized capture antibodies concentration at the detection zone to improve capture efficiency. Besides, the detection signal was further amplified by accumulated AuNP as the C. sakazakii labeled with AuNP probes was captured by antibodies conjugated with enhancer at the test line. We also studied the effect of different concentrations of capture antibodies and concentrated $\mathrm{AuNP}$ on detection performance, and found that $2.2 \mathrm{mg} / \mathrm{mL}$ of capture antibodies and $0.06 \mathrm{n} M$ concentrated AuNP were the optimal combination that could avoid a false-positive signal and maximally amplify the detection signal of the enhanced LFA. Using this strategy, the detection sensitivity of the enhanced LFA was $10^{3} \mathrm{cfu} / \mathrm{mL}$ and improved 100-fold compared with traditional LFA. The strip was highly specific to C. sakazakii, and the time for detection of C. sakazakii in PIF was shortened by $3 \mathrm{~h}$. In summary, the enhanced LFA developed by the addition of concentrated AuNP as the enhancer can be used as a sensitive, rapid, visual qualitative and point-of-care test method for detecting target analytes.
\end{abstract}

Key words: enhanced lateral flow immunoassay, gold nanoparticle, Cronobacter sakazakii, powdered infant formula

Received December 9, 2017

Accepted January 10, 2018.

${ }^{1}$ These authors contributed equally to this paper.

${ }^{2}$ Corresponding author: mcxwh2006@qq.com

\section{INTRODUCTION}

Cronobacter sakazakii, a member of the Cronobacter spp. (Joseph et al., 2012), is one of the most frequently reported foodborne pathogens, and it causes necrotizing enterocolitis, sepsis, and meningitis in infants as well as immuno-compromised populations (Healy et al., 2010). Contaminated powdered infant formula (PIF) has been identified as the most likely vehicle of transmission of the infection caused by C. sakazakii (Strydom et al., 2012; Li et al., 2014). Traditional microbiological methods take 5 to $7 \mathrm{~d}$ to complete analysis of $C$. sakazakii, and thus are time-consuming and laborious (Druggan et al., 2009). Currently, a variety of alternative techniques have been reported to detect C. sakazakii, including nucleic acid amplification methods (Zhou et al., 2016), ELISA (Kong et al., 2015), electrochemical biosensor, and immunomagnetic separation assay (Shukla et al., 2016). Although these methods are rapid and sensitive, most of them require laborious procedures, expensive equipment, and professional expertise. Such disadvantages impede these methods from on-site implementation. Thus, it is of great significance to develop a simple, sensitive, and rapid method to monitor C. sakazakii in PIF and prevent the risk of foodborne diseases caused by C. sakazakii.

Lateral flow immunoassay (LFA), which uses gold nanoparticles (AuNP) as the color marker with unique optical properties, extraordinary chemical stability, and binding capacity for biomolecules (Baptista et al., 2008), is a rapid, simple, and low-cost system for detection of target analytes in food, clinical diagnostics, and the environment with the naked eye (Tao et al., 2016). Nevertheless, the further application of LFA is impeded by poor sensitivity (Chen et al., 2015). To improve the sensitivity, researchers have replaced the traditional label-AuNP by AuNP nanocomposite and novel nanoparticle, including AuNP-decorated silica nanorods (Xu et al., 2014), quantum dots (Qu et al., 2016), fluorescence-quenching (Shen et al., 2017), and so on, which involved sophisticated preparation procedures and advanced detection tools. Meanwhile, the 
advances based on silver staining (Rodríguez et al., 2016), enzyme labeling (Cho and Irudayaraj, 2013), or $\mathrm{NH}_{2} \mathrm{OH} \cdot \mathrm{HCl}$ enlargement ( $\mathrm{Li}$ et al., 2013) have been widely used to amplify the detection signals; however, these methods rely on additional procedures to add extra reagents and extend the detection time (Chen et al., 2015). Therefore, it is necessary to significantly improve the sensitivity of traditional LFA by a simple and effective enhancing method.

One of the principal reasons for poor sensitivity of LFA is that the reaction is transient, caused by capillary action, which leads to low binding rate between the probe-labeled targets and the capture antibodies immobilized at the nitrocellulose (NC) membranes (Moghadam et al., 2015; Ren et al., 2016). Increasing the immobilized amount of capture antibodies can enhance the binding efficiency, but this methodology is ultimately limited by the protein binding capacity of NC membranes (Moghadam et al., 2015). Thus, the application of a novel strategy to overcome the limitation of protein binding capacity of $\mathrm{NC}$ membranes may improve the sensitivity of LFA.

Considering all aspects described above, the aim of present study was to increase the immobilized amount of capture antibodies to significantly improve the sensitivity of traditional LFA by a simple and effective method. Herein we proposed the enhanced LFA with concentrated AuNP as the enhancer to increase the immobilized amount of capture antibodies and amplify the signal for ultrasensitive detection of C. sakazakii in PIF.

\section{MATERIALS AND METHODS}

\section{Chemicals and Equipment}

Gold (III) chloride trihydrate $\left(\mathrm{HAuCl}_{4} \cdot 3 \mathrm{H}_{2} \mathrm{O}\right)$, sodium citrate $\left(\mathrm{C}_{6} \mathrm{H}_{5} \mathrm{Na}_{3} \mathrm{O}_{7} \cdot 2 \mathrm{H}_{2} \mathrm{O}\right)$, and $\mathrm{BSA}$ were obtained from Sigma (St. Louis, MO). The pair of anti- $C$. sakazakii monoclonal antibodies (2H1 as the detection antibody, 5C4 as the capture antibody) and goat antimouse IgG was purchased from Hangzhou Goodhere Biotechnology Co. Ltd. (Hangzhou, China). The sample pad, NC membrane, conjugate pad, absorption pad, and polyvinyl chloride pad used to fabricate the LFA were obtained from Hangzhou Goodhere Biotechnology Co. Ltd. Ponceau S was acquired from Beyotime Institute of Biotechnology (Shanghai, China). Other reagents used were of analytical purity.

The XYZ 3050 strip dispenser from BioDot (Irvine, CA) was used to spray the test (T) and control (C) line. A HGS201 cutter purchased from Autokun (Hangzhou, China) was used to cut the strips. A JH250Windows
AuNP-based strip reader supplied by Weifang Bainuo Di Biotechnology Co. Ltd. (Weifang, China) was used for measuring the signal of the $\mathrm{T}$ line. A SU-8010 high-resolution scanning electron microscope (Hitachi, Japan) was applied to observe the AuNP at the T line of the strip.

\section{Bacterial Strains}

Four C. sakazakii strains (ATCC 29544, ATCC 12868, ATCC 29004, ATCC BAA-894), 7 other Cronobacter spp., and 11 non-Cronobacter strains were used. All strains were cultured in nutrient broth for 12 to $16 \mathrm{~h}$ at $37^{\circ} \mathrm{C}$ shaking at $160 \mathrm{rpm}$.

\section{Synthesis of Detection Antibody Conjugated with AuNP}

The AuNP (average diameter $35 \mathrm{~nm}$ ) were synthesized according to previously reported methods (Frens, 1973). The synthesis procedures of anti-C. sakazakii monoclonal detection antibodies $(2 \mathrm{H} 1)$ conjugated with AuNP based on the following procedures constructed by our team (Zhao et al., 2017) were performed by adding $10 \mu \mathrm{g}$ of detection antibodies to $\mathrm{pH}$-adjusted 1-mL AuNP aliquots with $4 \mu \mathrm{L}$ of $0.2 \mathrm{M} \mathrm{K}_{2} \mathrm{CO}_{3}$ followed by incubation for $1 \mathrm{~h}$ at room temperature. Subsequently, $100 \mu \mathrm{L}$ of blocking solution (10\% BSA) was added and then incubated for another $1 \mathrm{~h}$ at room temperature to block the residual surface of AuNP. Then, the mixtures were centrifuged at $7,000 \times g$ for $20 \mathrm{~min}$ at $4^{\circ} \mathrm{C}$ to discard unbound antibodies, and the precipitate was re-dispersed in $100 \mu \mathrm{L}$ of $10 \mathrm{mM}$ Tris- $\mathrm{HCl}$ buffer containing $1 \%$ BSA, $2 \%$ trehalose, and $2 \mu \mathrm{L}$ of $10 \%$ BSA to increase flow closure. The obtained AuNP probes were stored at $4^{\circ} \mathrm{C}$ for further uses.

\section{Preparation of the Test-Line Solution for the Enhanced LFA}

Then, 35-nm AuNP were centrifuged at 7,000 $\times g$ for 20 min at $4^{\circ} \mathrm{C}$ and then the supernatant was removed. The pellet was suspended in $10 \mathrm{~m} M$ Tris- $\mathrm{HCl}$ buffer as concentrated AuNP solution, which was used as an enhancer conjugated with anti-C. sakazakii monoclonal capture antibodies $(5 \mathrm{C} 4)$, and incubated at $4^{\circ} \mathrm{C}$ for 1 h. After the incubation, the entire solution was used as the test-line solution to spot onto the $\mathrm{T}$ lines of enhanced LFA. To evaluate the effect of concentration of AuNP and capture antibodies on detection performance, AuNP and capture antibodies at different concentrations were optimized to construct the enhanced LFA and this experiment was performed in triplicate. 


\section{Fabrication of LFA}

The assembly of traditional LFA was performed as shown in Figure 1A. The sample pad made from glass fiber membrane was pretreated with running buffer (10 $\mathrm{m} M$ Tris- $\mathrm{HCl}, 0.2 \%$ BSA, $0.2 \%$ polyvinyl pyrrolidone, $0.05 \%$ casein, $0.5 \%$ Tween 20 ), and then dried at $42^{\circ} \mathrm{C}$ for $1 \mathrm{~h}$. The conjugate pad, which consisted of glass fiber membrane, was soaked in the AuNP-conjugated detection antibodies described above, and dried at $37^{\circ} \mathrm{C}$ for $1 \mathrm{~h}$. The anti-C. sakazakii monoclonal capture antibodies $(5 \mathrm{C} 4)$ and goat anti-mouse $\operatorname{IgG}(0.6 \mathrm{mg} / \mathrm{mL})$ were respectively immobilized onto the $\mathrm{NC}$ membranes as $\mathrm{T}$ and $\mathrm{C}$ lines at a sprayed speed of $1 \mu \mathrm{L} / \mathrm{cm}$ followed by drying at $37^{\circ} \mathrm{C}$ for $1 \mathrm{~h}$. The sample pad, NC membrane, conjugate pad, absorption pad, and polyvinyl chloride pad were assembled as the strip.

The schematic of the enhanced LFA developed in this context is displayed in Figure 1B. The preparation procedures were similar to traditional LFA, except that the capture anti-C. sakazakii monoclonal antibodies (5C4) were replaced by concentrated AuNP solution conjugated with capture antibodies (above-mentioned $\mathrm{T}$ line solution).

\section{Ponceau S Staining}

The $\mathrm{T}$ line introduced $2.2 \mathrm{mg} / \mathrm{mL}$ of capture antibodies conjugated with $0.06 \mathrm{nM}$ concentrated AuNP of the enhanced strip, and the $\mathrm{T}$ line with $2.2 \mathrm{mg} /$ $\mathrm{mL}$ of capture antibodies of the traditional strip was respectively stained with Ponceau $\mathrm{S}$ to detect immobilized protein of the $\mathrm{T}$ line. The $\mathrm{T}$ line of strip was washed with water to remove the unbound antibodies and then stained with Ponceau S and agitated for 10 min until the bound protein could be clearly observed (Wang et al., 2013). Subsequently, the membrane was gently washed with water to remove the excess Ponceau S solution.

\section{Sensitivity Test of LFA}

The 10-fold series dilutions of C. sakazakii were prepared in $0.85 \%$ sodium chloride followed by dripping $100 \mu \mathrm{L}$ of dilutions onto the sample pad of the traditional LFA and enhanced LFA (the T line solution was $2.2 \mathrm{mg} / \mathrm{mL}$ of capture antibodies conjugated with 0.06 $\mathrm{n} M$ concentrated AuNP), respectively, for the sensitivity test. After $15 \mathrm{~min}$, the signal strength of the strip

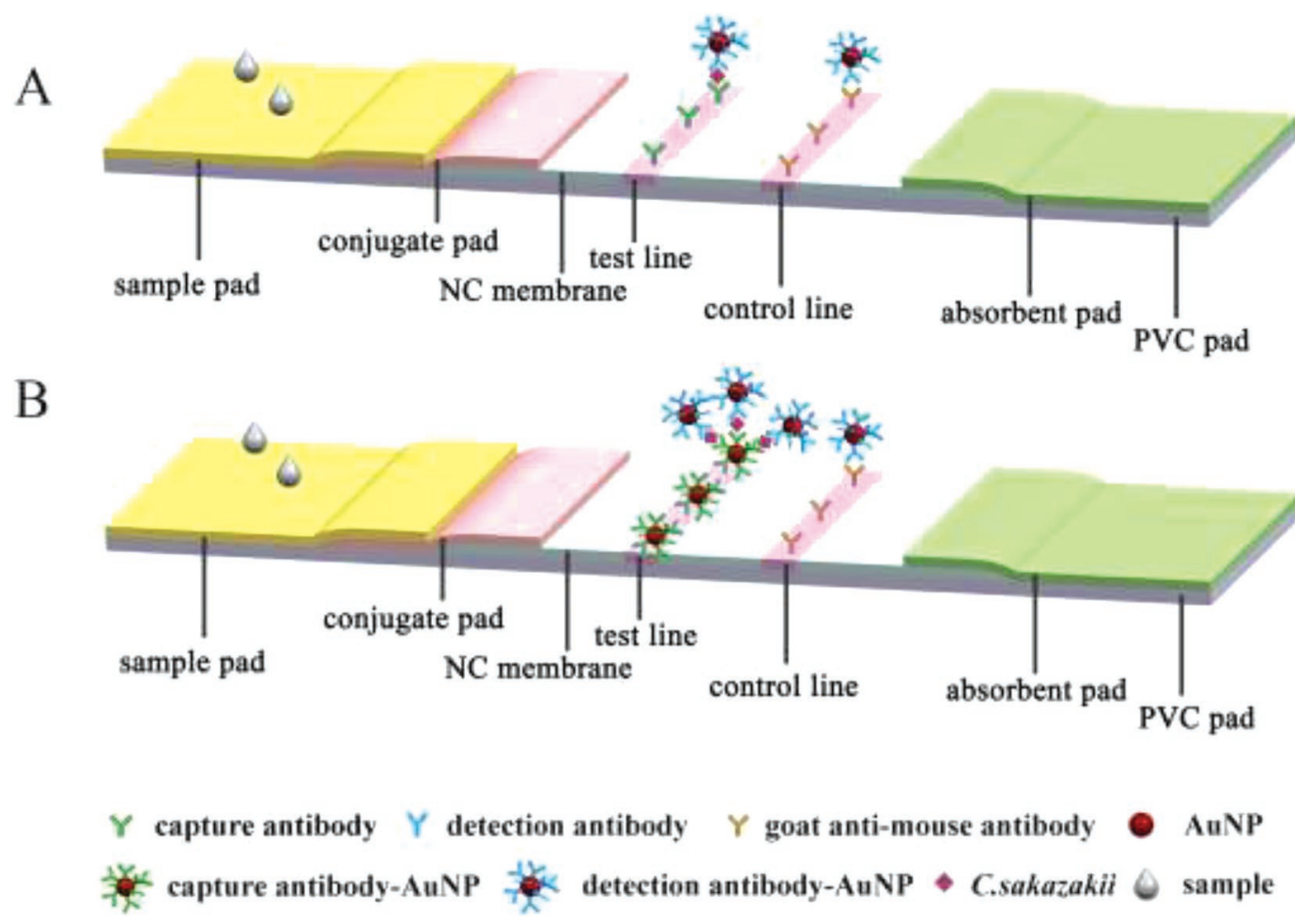

Figure 1. Schematic illustration of the lateral flow immunoassay (LFA) for detection of Cronobacter sakazakii. (A) Traditional LFA, (B) enhanced LFA. NC = nitrocellulose; AuNP = gold nanoparticle; PVC = polyvinyl chloride. Color version available online. 
was recorded by the strip reader. Sensitivity test was performed in triplicate.

\section{Specificity Test of LFA}

Cronobacter sakazakii (ATCC 29544, ATCC 29004, ATCC 12868, ATCC BAA-894), other Cronobacter spp., and non-Cronobacter strains with a concentration of $10^{7} \mathrm{cfu} / \mathrm{mL}$ were applied to assess the specificity of the traditional LFA and enhanced LFA (the T line solution was $2.2 \mathrm{mg} / \mathrm{mL}$ of capture antibodies conjugated with $0.06 \mathrm{n} M$ concentrated AuNP). The specificity test was performed in triplicate.

\section{Detection of C. sakazakii in PIF}

Ten grams each of non- $C$. sakazakii PIF sample was homogenized in $90 \mathrm{~mL}$ of nutrient broth medium and C. sakazakii (6 cfu), followed by incubation at $37^{\circ} \mathrm{C}$ and shaking at $160 \mathrm{rpm}$ (Wang et al., 2015; Shukla et al., 2016). Aliquots of $1 \mathrm{~mL}$ from each bottle at 3, 4.5, $6,7.5$, and $9 \mathrm{~h}$ incubation periods were collected. One milliliter of sample culture solutions was centrifuged at $5,000 \times g$ for $5 \mathrm{~min}$ at $4^{\circ} \mathrm{C}$ to remove the lipid layer. To remove the proteins and lipids more completely, the solutions were suspended and re-centrifuged at 5,000 $\times g$ for 5 min at $4^{\circ} \mathrm{C}$ (Kim et al., 2017). The obtained skim milk samples were detected by the traditional LFA and enhanced LFA (the T line solution was 2.2 $\mathrm{mg} / \mathrm{mL}$ of capture antibodies conjugated with $0.06 \mathrm{nM}$ concentrated AuNP).

\section{RESULTS AND DISCUSSION}

\section{Effect of Capture Antibodies Concentrations on the Sensitivity of Traditional LFA}

The concentration of capture antibodies $(5 \mathrm{C} 4)$ at the $\mathrm{T}$ line, interacted with labeled $C$. sakazakii, is a pivotal factor to affect the sensitivity of the LFA. The concentrations of capture antibodies ranging from 0.6 to $1.8 \mathrm{mg} / \mathrm{mL}$ were optimized to determine the optimal protein concentration and obtain the strongest detection signal of traditional LFA. As illustrated by Figure 2 , the signal intensity of the $\mathrm{T}$ line was enhanced with the increase of capture reagent concentration until reaching the highest value at $1.4 \mathrm{mg} / \mathrm{mL}$ and obviously decreased at $1.8 \mathrm{mg} / \mathrm{mL}$. The trend gained in this study was consistent with the results found by Chen et al. (2016). These results might be caused by the limited protein binding capacity of NC membranes. The extra capture antibodies that were free at the $\mathrm{NC}$ membranes might also combine with AuNP probe-labeled C. sakazakii and take away some color signals under

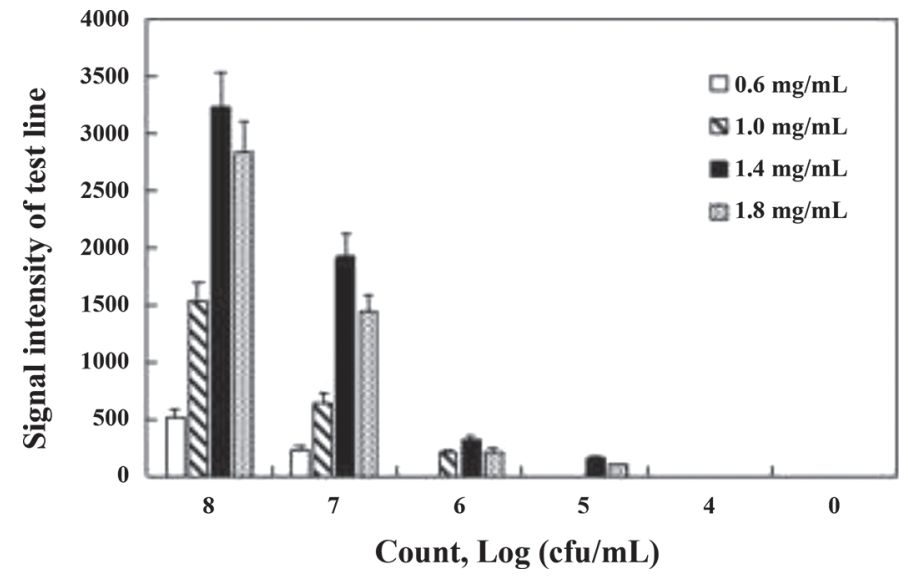

Figure 2. Effect of capture antibody concentrations on the detection of Cronobacter sakazakii by traditional lateral flow immunoassay. Error bars represent SD.

chromatographic flow; hence, excessive capture antibodies exceeding the protein binding capacity of $\mathrm{NC}$ membranes contrarily decreased the detection signal of the strips. Consequently, the optimal capture antibodies concentration of traditional LFA was $1.4 \mathrm{mg} / \mathrm{mL}$ and the detection limit was $10^{5} \mathrm{cfu} / \mathrm{mL}$.

\section{Principle of the Enhanced LFA}

The efficient and simple strategy for signal enhancement was based on the increasing concentration of bound protein at $\mathrm{NC}$ membranes to improve reaction rate (Figure 1), which employed the high surface areas and biomolecules binding capacity of AuNP (Baptista et al., 2008; Lai et al., 2010). As well known, increasing the density of capture reagents could improve the binding rate to enhance the sensitivity of the strip; however, the method was eventually impeded by the protein binding capacity of the NC membrane (Moghadam et al., 2015), as can be seen in Figure 2. In our strategy, 2 kinds of AuNP were used: one as the typical marker, and the other, concentrated AuNP, as the novel enhancer to conjugate with capture antibodies that could provide additional sites to bind more protein. The capture antibodies could conjugate with the enhancer through valence combination, electrostatic and hydrophobic forces, and then immobilized at the $\mathrm{T}$ line of the NC membrane. This led to an increase in the number of capture antibodies immobilized at the $\mathrm{T}$ lines and the reaction rate between capture antibodies and C. sakazakii labeled with AuNP probes. The increased reaction rate improved the amount of labeled C. sakazakii fixed at the detection area and led to a stronger signal. Apart from that, a complex was formed consisting of target antigen labeled with AuNP probes 
Table 1. Effect of capture antibodies and concentrated gold nanoparticle (AuNP) concentrations on the detection of Cronobacter sakazakii by the enhanced lateral flow immunoassay; the results of signal intensity of test line were indicated by the mean $\pm \mathrm{CV}$

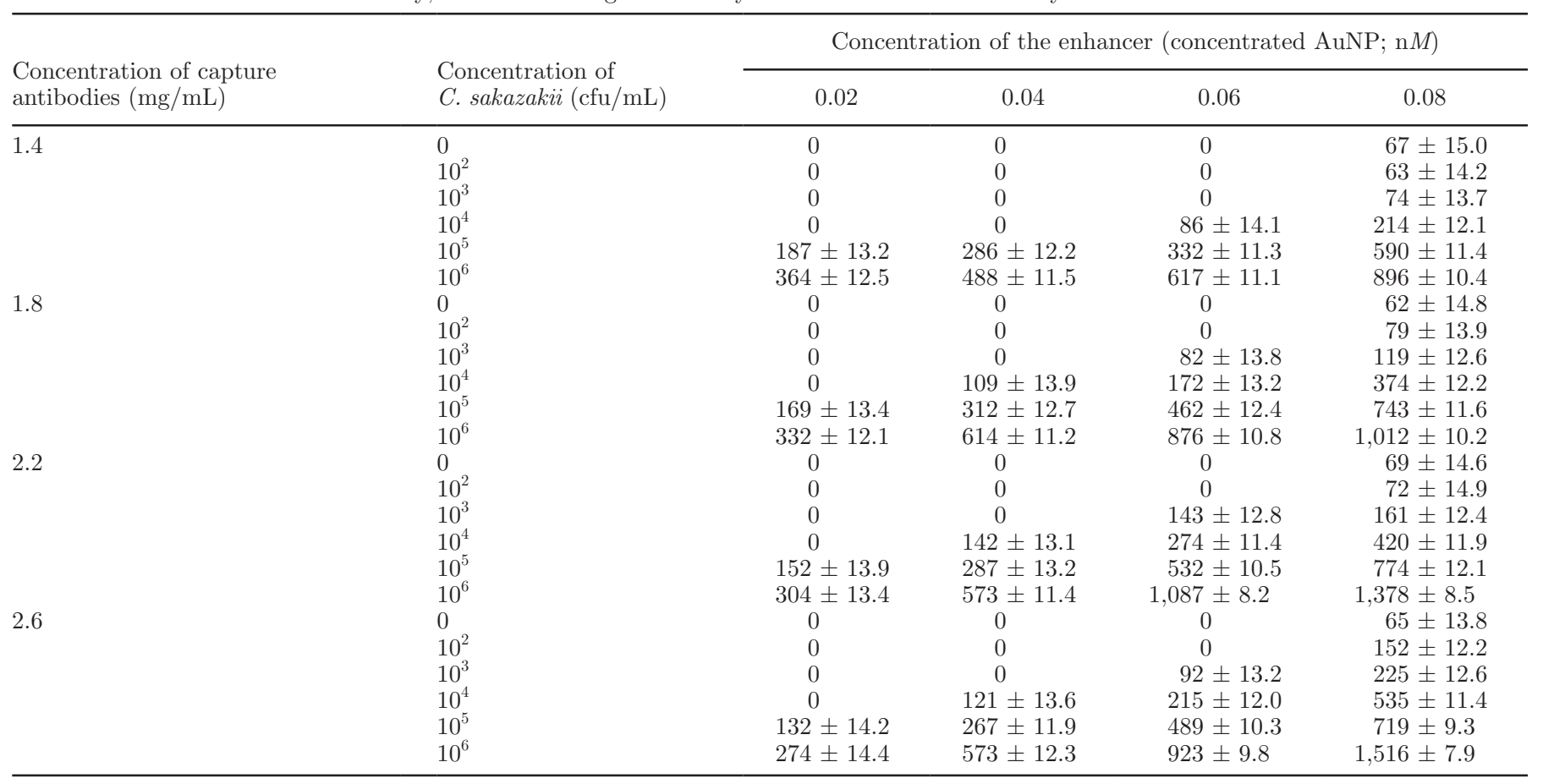

and capture antibodies conjugated with AuNP through sandwich-assay immunoreactions. The AuNP were aggregated in a small area at the detection zone. Thus, detection signals were further enhanced.

\section{Optimal Conditions for the Enhanced LFA}

In this study, concentrated AuNP were deployed to increase the amount of capture antibodies coated on the NC membranes, and AuNP themselves played a role in enhancing the detection signal because of its optical property. Thus, the concentrations of capture antibodies and concentrated AuNP were optimized systematically to avoid the false-positive signal and to improve the sensitivity to a great extent. The detection limits of LFA and the intensity of the T line were used to evaluate the analytical performance.

Table 1 shows the effect of different concentrations of capture antibodies (5C4) and concentrated AuNP on the detection performance. Only a small enhancement occurred at a low concentration of $0.02 \mathrm{n} M$ AuNP, mainly related to the limited protein binding capacity of low-concentration AuNP. When the concentration of AuNP was 0.04 and $0.06 \mathrm{nM}$, the strongest detection signal was obtained with 1.8 and $2.2 \mathrm{mg} / \mathrm{mL}$ of capture antibodies immobilized on the $\mathrm{NC}$ membranes, corresponding to a sensitivity of $10^{4}$ and $10^{3} \mathrm{cfu} / \mathrm{mL}$, respectively. Although the sensitivity and the number of bound protein on the NC members were higher than others at the concentration of $0.08 \mathrm{n} M$ AuNP, the high concentration of AuNP induced the false-positive result. Hence, the most suitable concentration of AuNP of the enhanced strip was $0.06 \mathrm{n} M$, which led to the concentration of bound capture antibodies on the $\mathrm{NC}$ membranes increasing from 1.4 to $2.2 \mathrm{mg} / \mathrm{mL}$ and reducing the detection limit to $10^{3} \mathrm{cfu} / \mathrm{mL}$ in comparison to traditional LFA.

To show the influence of the capture antibodies concentration on the densities of $\mathrm{T}$ line in the enhancement assay, the $0.06 \mathrm{n} M$ concentrated AuNP was used as an example, as shown in Figure 3. The signal intensity at the $\mathrm{T}$ line increased up to $2.2 \mathrm{mg} / \mathrm{mL}$, and a further concentration increase caused a decrease in the signal intensity. This result was explained by the fact that the addition of the enhancer increased the amount of bound protein based on the biomolecule binding capacity of AuNP. However, excessive capture antibodies that exceeded the protein binding capacity of concentrated AuNP added and NC membranes would result in signal loss. These phenomena also demonstrated that different optimal concentrations of capture antibodies corresponded to the addition of different concentrations of AuNP.

The $1.4 \mathrm{mg} / \mathrm{mL}$ capture antibodies were taken as an example to clearly illustrate the effect of the concentration of AuNP on the signal intensity of $\mathrm{T}$ lines, as 


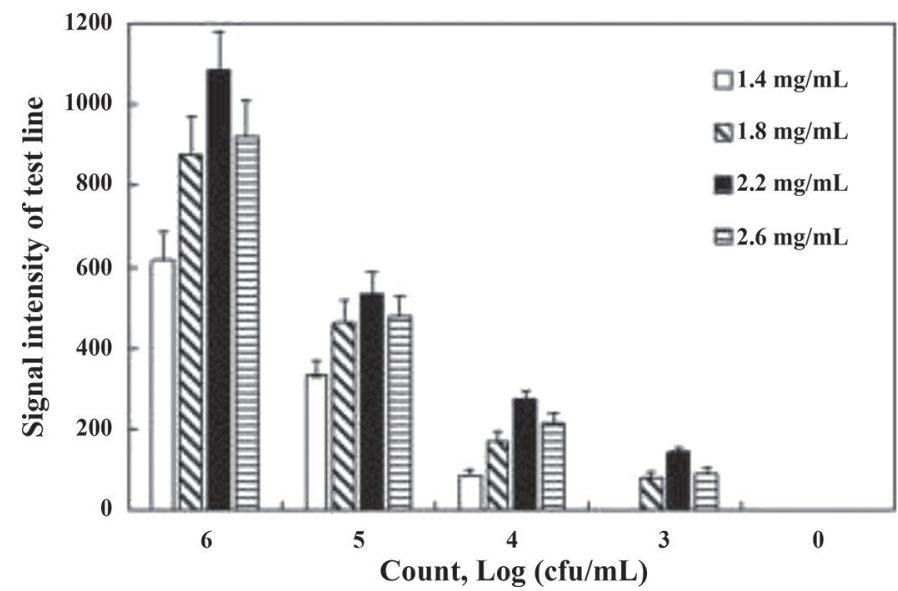

Figure 3. Effect of capture antibodies concentrations on the detection of Cronobacter sakazakii by enhanced lateral flow immunoassay at a concentration of $0.06 \mathrm{n} M$ gold nanoparticles. Error bars represent SD.

shown in detail in Figure 4. The signal intensity of the $\mathrm{T}$ lines was enhanced with the increase of the AuNP concentration (Figure 4), due to the optical property of AuNP and the fact that the increase of the AuNP concentration elicited the increase in concentration of bound protein (Table 1). However, the concentration of AuNP increased up to $0.08 \mathrm{n} M$, resulting in a falsepositive result. Therefore, it was important to select the most suitable concentration of AuNP to avoid background signal and maximally amplify the detection signal of enhanced LFA.

In general, the enhanced LFA became more sensitive as an increase in the concentration of captured reagents immobilized at the detection zone by the efficient application of concentrated AuNP. Also, the addition of concentrated AuNP provided additional binding sites

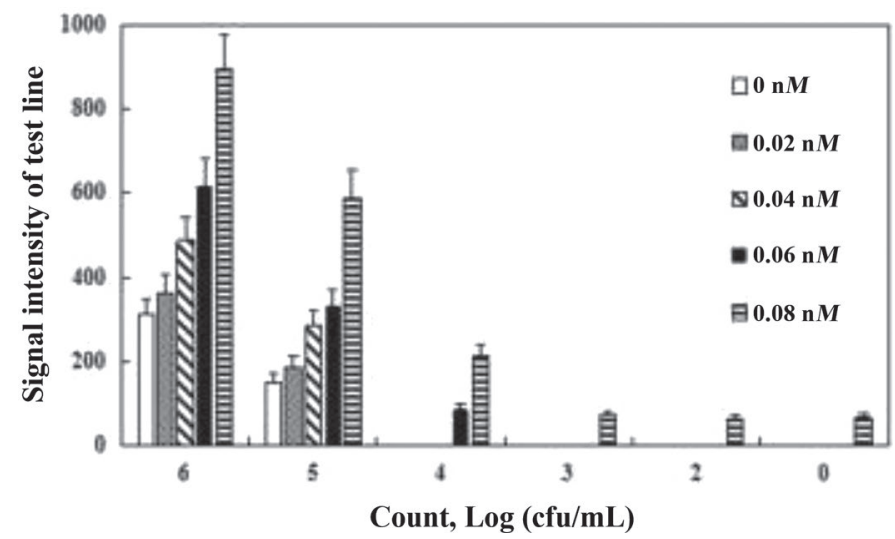

Figure 4. Effect of concentrated gold nanoparticle concentrations on the detection of Cronobacter sakazakii by enhanced lateral flow immunoassay at a concentration of $1.4 \mathrm{mg} / \mathrm{mL}$ of capture antibodies. Error bars represent SD. for captured antibodies, which overcame the problem of limited protein binding ability of the NC membrane to a certain extent.

\section{Evaluation of the Enhanced LFA}

Scanning Electron Microscopy and Protein Staining. First, the concentrated AuNP (0.06 nM) used to conjugate with capture antibodies $(2.2 \mathrm{mg} /$ $\mathrm{mL}$ ) immobilized on the NC membranes was checked through scanning electron microscopy. The concentrated AuNP were distributed on the $\mathrm{T}$ line in a dispersed manner (Figure 5A). In addition, AuNP maintained high and long-term stability in solid state attributed to the high strength provided by the combination of antibodies and AuNP (Chandler et al., 2000). The Ponceau $\mathrm{S}$ was applied to stain the bound protein on the $\mathrm{T}$ line of $\mathrm{NC}$ membranes. Comparing Figure 5B (1) and (2) showed that the color intensity at the $\mathrm{T}$ line of the enhanced strip $(2.2 \mathrm{mg} / \mathrm{mL}$ of capture antibodies conjugated with $0.06 \mathrm{nM}$ concentrated AuNP) was much deeper than that of traditional strip with $2.2 \mathrm{mg} / \mathrm{mL}$ of capture antibodies, indicating that the addition of enhancer-concentrated AuNP increased the amount of bound capture antibodies at the $\mathrm{T}$ lines. These findings were consistent with the proposed enhancing principle.

Comparison of Sensitivity Between Traditional LFA and Enhanced LFA. As indicated in Figure 6 , the detection limit of enhanced LFA under optimum conditions was as low as $10^{3} \mathrm{cfu} / \mathrm{mL}$, whereas that of the traditional LFA was $10^{5} \mathrm{cfu} / \mathrm{mL}$, determined by the naked eye and a strip reader, indicating that the enhanced LFA was 100-fold more sensitive than the traditional LFA. Moreover, the color intensity of $\mathrm{T}$ line of enhancement assay was deeper than that of the traditional assay, which was beneficial for reading by the strip reader (Rivas et al., 2015). In comparison to other

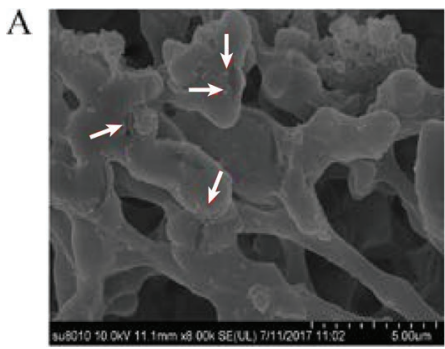

B

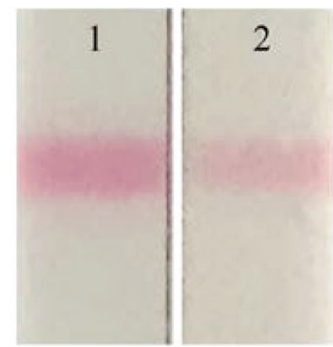

Figure 5. Evaluation of the enhanced lateral flow immunoassay. (A) Scanning electron microscopy images of the test (T) line of the nitrocellulose-enhanced strip after fabrication of the enhanced strip arrows indicated the position with the presence of gold nanoparticles. (B) Protein staining of the $\mathrm{T}$ line of nitrocellulose strip after fabrication of the strip: (1) enhanced strip and (2) traditional strip. Color version available online. 

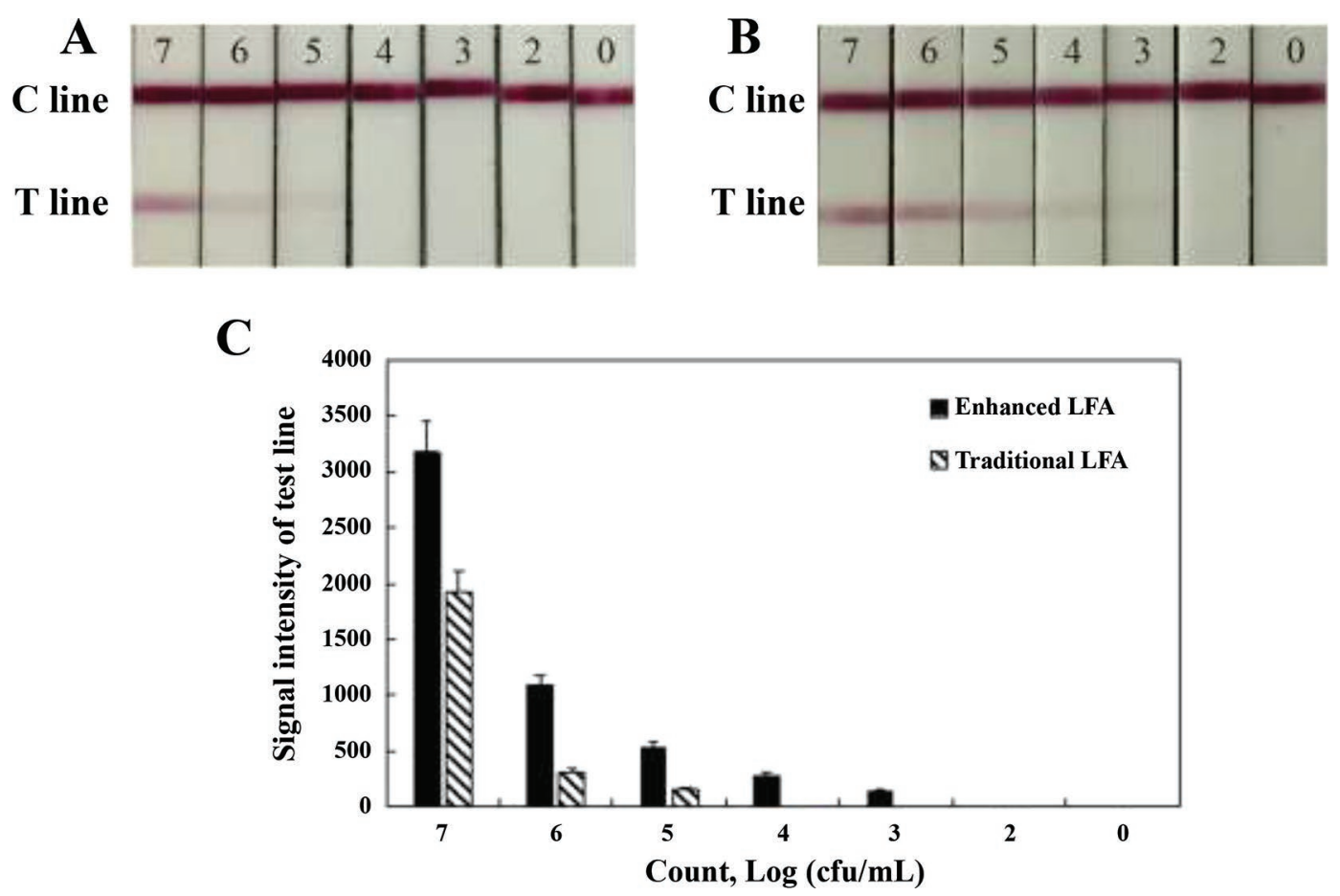

Figure 6. Comparison of sensitivity between traditional lateral flow immunoassay (LFA) and enhanced LFA. (A) Traditional LFA, (B) enhanced LFA, (C) traditional LFA and enhanced LFA. The concentrations of Cronobacter sakazakii were $10^{7}, 10^{6}, 10^{5}, 10^{4}, 10^{3}$, and $10^{2}$ cfu $/ \mathrm{mL}^{2}$ from 7 to 2 , respectively, and 0 represented $0 \mathrm{cfu} / \mathrm{mL}$. C line $=$ control line; $\mathrm{T}$ line $=$ test line. Error bars represent SD. Color version available online.

Table 2. Results of specificity test of lateral flow immunoassay (LFA)

\begin{tabular}{|c|c|c|c|}
\hline \multirow[b]{2}{*}{ No. } & \multirow[b]{2}{*}{ Strain $^{1}$} & \multicolumn{2}{|c|}{ Results $^{2}$} \\
\hline & & Traditional LFA & Enhanced LFA \\
\hline 1 & Cronobacter sakazakii ATCC 29544 & + & + \\
\hline 2 & Cronobacter sakazakii ATCC 128868 & + & + \\
\hline 3 & Cronobacter sakazakii ATCC 29004 & + & + \\
\hline 4 & Cronobacter sakazakii ATCC BAA-894 & + & + \\
\hline 5 & Cronobacter malonaticus DSM 18702 & - & - \\
\hline 6 & Cronobacter turicensis DSM 18703 & - & - \\
\hline 7 & Cronobacter muytjensii ATCC 51329 & - & - \\
\hline 8 & Cronobacter universalis NCTC 9529 & - & - \\
\hline 9 & Cronobacter dublinensis DSM 18705 & - & - \\
\hline 10 & Cronobacter dublinensis ssp. lausannensis DSM 18706 & - & - \\
\hline 11 & Cronobacter dublinensis ssp. lactaridi DSM 18707 & - & - \\
\hline 12 & Citrobacter freundii ATCC 10787 & - & - \\
\hline 13 & Salmonella Enteritidis CICC 21482 & - & - \\
\hline 14 & Escherichia coli ATCC 43895 & - & - \\
\hline 15 & Shigella flexneri CMCC 51572 & - & - \\
\hline 16 & Serratia marcescens ATCC 8100 & - & - \\
\hline 17 & Enterobacter cloacae ATCC 13048 & - & - \\
\hline 18 & Proteus mirabilis ATCC 12453 & - & - \\
\hline 19 & Klebsiella pneumonia ATCC 27336 & - & - \\
\hline 20 & Staphylococcus aureus ATCC 13565 & + & + \\
\hline 21 & Listeria monocytogenes CMCC 54004 & - & - \\
\hline 22 & Enterobacter aerogenes ATCC 13048 & - & - \\
\hline
\end{tabular}

${ }^{1}$ ATCC $=$ American Type Culture Collection; DSM = Deutsche Sammlung von Mikroorganismen und Zellkulturen; NCTC = National Collection of Type Cultures; CICC = China Center of Industrial Culture Collection; CMCC $=$ National Center for Medical Culture Collections.

${ }^{2}+=$ positive results; $-=$ negative results. 
Table 3. Results of detection of Cronobacter sakazakii in powdered infant formula

\begin{tabular}{|c|c|c|c|c|}
\hline Enrichment time (h) & \multicolumn{2}{|c|}{ Traditional LFA ${ }^{1}$} & \multicolumn{2}{|c|}{ Enhanced LFA } \\
\hline 3.0 & - & - & - & - \\
\hline 6.0 & - & - & + & - \\
\hline 7.5 & - & - & + & - \\
\hline 9.0 & + & - & + & - \\
\hline
\end{tabular}

${ }^{1} \mathrm{LFA}=$ lateral flow immunoassay $+=$ positive results; $-=$ negative results.

methods of signal enhancement such as dual labeling of AuNP (Zhong et al., 2016), enzyme labeling (Cho and Irudayaraj, 2013), and fluorescent microspheres (Xie et al., 2014), our enhancing method was simple, fast, and extremely suitable for on-site detection, because the reagent used to improve the reaction rate was already part of the traditional strips and extra detection procedures were not needed. In addition, the detection results could be achieved within $15 \mathrm{~min}$ and seen by the naked eye and no extra instruments were required.

\section{Specificity Test of LFA}

Some pathogens with close relative and similar antigenic sites to C. sakazakii, common foodborne pathogens, and C. sakazakii were used to examine the specificity of the traditional method and the enhanced method. To ensure the specificity of the strip, monoclonal antibodies were used instead of polyclonal antibodies at the $\mathrm{T}$ line (Li et al., 2011). As listed in Table 2, 4 strains of C. sakazakii showed positive results and the 18 non-C. sakazakii strains showed negative results with the exception of Staphylococcus aureus. The crossreactivity with $S$. aureus was also found in the previous reports (Xiong et al., 2014; Xia et al., 2016). It was related to a covalently bound protein called protein A in the cell wall of $S$. aureus, which has a high affinity with the Fc region of IgG (Xiong et al., 2014). These findings demonstrated that the LFA developed in our study was specific to C. sakazakii.

\section{Detection of C. sakazakii in PIF}

As for detection of C. sakazakii in PIF by the LFA, the proteins and lipids should be removed in advance to avoid the matrix effect (Naik et al., 2017). As indicated in Table 3, the enrichment time for detecting $C$. sakazakii (6 cfu/10 g) in PIF by the traditional LFA and enhanced LFA was 9 and $6 \mathrm{~h}$, respectively. It was obviously indicated that the detection time could be highly shortened by using enhanced LFA.

\section{CONCLUSIONS}

In conclusion, an optimized enhanced LFA using concentrated AuNP as enhancer was successfully developed to detect C. sakazakii in PIF. We have overcome the limitation of protein binding capacity of NC membranes to improve sensitivity using concentrated AuNP as the enhancer. By using this strategy, the concentration of capture antibodies immobilized at the $\mathrm{T}$ line was increased, and the number of AuNP accumulated was enhanced through sandwich-assay immunoreactions, leading to a 100 -fold improvement in sensitivity. No extra reagents or sophisticated instruments were required for this method. Thus, the enhanced LFA developed here is extremely simple, sensitive, and rapid and has the potential to be widely implemented in detection fields.

\section{ACKNOWLEDGMENTS}

This work was supported by the National High-Level Talents Special Support Program of China (Harbin) and "Academic Backbone" Project of Northeast Agricultural University (15XG26, Harbin, China).

\section{REFERENCES}

Baptista, P., E. Pereira, P. Eaton, G. Doria, A. Miranda, I. Gomes, P. Quaresma, and R. Franco. 2008. Gold nanoparticles for the development of clinical diagnosis methods. Anal. Bioanal. Chem. 391:943-950.

Chandler, J., T. Gurmin, and N. Robinson. 2000. The place of gold in rapid tests. IVD Technol. 6:37-49.

Chen, M., Z. Yu, D. Liu, T. Peng, K. Liu, S. Wang, Y. Xiong, H. Wei, H. Xu, and W. Lai. 2015. Dual gold nanoparticle lateflow immunoassay for sensitive detection of Escherichia coli O157:H7. Anal. Chim. Acta 876:71.

Chen, Y., C. Nan, Y. Xu, K. Huang, Y. Luo, and W. Xu. 2016. Pointof-care and visual detection of $P$. aeruginosa and its toxin genes by multiple LAMP and lateral flow nucleic acid biosens. Biosens. Bioelectron. 81:317-323.

Cho, I. H., and J. Irudayaraj. 2013. Lateral-flow enzyme immunoconcentration for rapid detection of Listeria monocytogenes. Anal. Bioanal. Chem. 405:3313-3319.

Druggan, P., C. Iversen, C. Iversen, and S. Fanning. 2009. Culture media for the isolation of Cronobacter spp. Int. J. Food Microbiol. 136:169-178. 
Frens, G. 1973. Controlled nucleation for the regulation of the particle size in monodisperse gold suspensions. Nature 241:20-22.

Healy, B., S. Cooney, S. O'Brien, C. Iversen, P. Whyte, J. Nally, J. J. Callanan, and S. Fanning. 2010. Cronobacter (Enterobacter sakazakii): An opportunistic foodborne pathogen. Foodborne Pathog. Dis. 7:339-350.

Joseph, S., H. Sonbol, S. Hariri, P. Desai, M. Mcclelland, and S. J. Forsythe. 2012. Diversity of the Cronobacter genus as revealed by multilocus sequence typing. J. Clin. Microbiol. 50:3031-3039.

Kim, H. S., Y. J. Kim, J. W. Chon, D. H. Kim, J. H. Yim, H. Kim, and K. H. Seo. 2017. Two-stage label-free aptasensing platform for rapid detection of Cronobacter sakazakii in powdered infant formula. Sensor. Actuat. B Chem. 239:94-99.

Kong, D., L. Liu, C. Xing, H. Kuang, and C. Xu. 2015. Sensitive and highly specific detection of Cronobacter sakazakii based on monoclonal sandwich ELISA. Food Agric. Immunol. 26:566-576.

Lai, C., G. M. Zeng, D. L. Huang, C. L. Feng, H. U. Shuang, S. U. Feng-Feng, M. H. Zhao, C. Huang, and Z. Wei. 2010. Detection based on immunogold labeling technique and its expected application in composting. Chin. J. Anal. Chem. 38:909-914.

Li, J., M. Zou, C. Yan, X. Qiang, Z. Fan, B. Li, Y. Wang, X. Qi, and Y. Yi. 2013. Gold immunochromatographic strips for enhanced detection of avian influenza and Newcastle disease viruses. Anal. Chim. Acta 782:54.

Li, X. S., F. Fu, Y. K. Lang, H. Z. Li, W. Wang, X. Chen, H. B. Tian, Y. J. Zhou, G. Z. Tong, and X. Li. 2011. Development and preliminary application of an immunochromatographic strip for rapid detection of infection with porcine reproductive and respiratory syndrome virus in swine. J. Virol. Methods 176:46-52.

Li, Y., Q. Chen, J. Zhao, H. Jiang, F. Lu, X. Bie, and Z. Lu. 2014. Isolation, identification and antimicrobial resistance of Cronobacter spp. isolated from various foods in China. Food Control 37:109-114.

Moghadam, B. Y., K. T. Connelly, and J. D. Posner. 2015. Two orders of magnitude improvement in detection limit of lateral flow assays using isotachophoresis. Anal. Chem. 87:1009-1017.

Naik, L., R. Sharma, B. Mann, K. Lata, Y. S. Rajput, and N. B. Surendra. 2017. Rapid screening test for detection of oxytetracycline residues in milk using lateral flow assay. Food Chem. 219:85-92.

Qu, H., Y. Zhang, B. Qu, H. Kong, G. Qin, S. Liu, J. Cheng, Q. Wang, and Y. Zhao. 2016. Rapid lateral-flow immunoassay for the quantum dot-based detection of puerarin. Biosens. Bioelectron. 81:358.

Ren, W., I. H. Cho, Z. Zhou, and J. Irudayaraj. 2016. Ultrasensitive detection of microbial cells using magnetic focus enhanced lateral flow sensors. Chem. Commun. (Camb.) 52:4930.

Rivas, L., A. D. L. Escosura-Muñiz, L. Serrano, L. Altet, O. Francino, A. Sánchez, and A. Merkoçi. 2015. Triple lines gold nanoparticlebased lateral flow assay for enhanced and simultaneous detection of Leishmania DNA and endogenous control. Nano Res. 8:37043714 .

Rodríguez, M. O., L. B. Covián, A. C. García, and M. C. BlancoLópez. 2016. Silver and gold enhancement methods for lateral flow immunoassays. Talanta 148:272-278.
Shen, H., S. Zhang, Q. Fu, W. Xiao, S. Wang, S. Yu, M. Xiao, H. Bian, and Y. Tang. 2017. A membrane-based fluorescence-quenching immunochromatographic sensor for the rapid detection of tetrodotoxin. Food Control. 81:101-106.

Shukla, S., G. Lee, X. Song, S. Park, and M. Kim. 2016. Immunoliposome-based immunomagnetic concentration and separation assay for rapid detection of Cronobacter sakazakii. Biosens. Bioelectron. $77: 986$.

Strydom, A., D. M. Cawthorn, M. Cameron, and R. C. Witthuhn. 2012. Species of Cronobacter-A review of recent advances in the genus and their significance in infant formula milk. Int. Dairy J. $27: 3-12$.

Tao, C., Q. Zhang, F. Na, D. Shi, and L. Bang. 2016. Development of a colloidal gold immunochromatographic strip assay for simple and fast detection of human $\alpha$-lactalbumin in genetically modified cow milk. J. Dairy Sci. 99:1773-1779.

Wang, J.-Y., M.-H. Chen, Z.-C. Sheng, D.-F. Liu, S.-S. Wu, and W.-H. Lai. 2015. Development of colloidal gold immunochromatographic signal-amplifying system for ultrasensitive detection of Escherichio coli O157:H7 in milk. RSC Advances 5:62300-62305.

Wang, X., H. Lin, J. Sui, and L. Cao. 2013. The effect of fish matrix on the enzyme-linked immunosorbent assay of antibiotics. J. Sci. Food Agric. 93:1603

Xia, S., Z. Yu, D. Liu, C. Xu, and W. Lai. 2016. Developing a novel immunochromatographic test strip with gold magnetic bifunctional nanobeads (GMBN) for efficient detection of Salmonella choleraesuis in milk. Food Control 59:507-512.

Xie, Q. Y., Y. H. Wu, Q. R. Xiong, H. Y. Xu, Y. H. Xiong, K. Liu, Y. Jin, and W. H. Lai. 2014. Advantages of fluorescent microspheres compared with colloidal gold as a label in immunochromatographic lateral flow assays. Biosens. Bioelectron. 54:262-265.

Xiong, Q., X. Cui, J. K. Saini, D. Liu, S. Shan, Y. Jin, and W. Lai 2014. Development of an immunomagnetic separation method for efficient enrichment of Escherichia coli O157:H7. Food Control $37: 41-45$

Xu, H., J. Chen, J. Birrenkott, J. X. Zhao, S. Takalkar, K. Baryeh, and G. Liu. 2014. Gold-nanoparticle-decorated silica nanorods for sensitive visual detection of proteins. Anal. Chem. 86:7351-7359.

Zhao, Y., X. Jiang, Y. Qu, R. Pan, X. Pang, Y. Jiang, and C. Man. 2017. Salmonella detection in powdered dairy products using a novel molecular tool. J. Dairy Sci. 100:3480-3496.

Zhong, Y., Y. Chen, L. Yao, D. Zhao, L. Zheng, G. Liu, Y. Ye, and W. Chen. 2016. Gold nanoparticles based lateral flow immunoassay with largely amplified sensitivity for rapid melamine screening. Mikrochim. Acta 183:1989-1994

Zhou, B., B. Chen, W. Xin, L. Fan, Y. Pei, Z. P. Aguilar, W. Hua, and H. Xu. 2016. A new application of a sodium deoxycholatepropidium monoazide-quantitative PCR assay for rapid and sensitive detection of viable Cronobacter sakazakii in powdered infant formula. J. Dairy Sci. 99:9550-9559. 